\title{
OBSERVABLE MEDIUM EFFECTS FOR ATMOSPHERIC NEUTRINOS
}

\author{
J. Bernabéu ${ }^{* \dagger}$ and S. Palomares-Ruiz \\ Departamento de Física Teòrica, Universitat de València.
}

Abstract: We discuss the possibility to observe matter effects in atmospheric neutrino oscillations. The main conclusion is that an impact on the $\nu_{\mu}$ survival probability requires the action of the MSW resonance, which becomes visible for baselines above $\sim 7000 \mathrm{~km}$. The associated muon charge asymmetry carries information on $\theta_{13}$ and the sign of $\Delta m_{31}^{2}$.

\section{Introduction}

Present evidence for neutrino masses and mixings can be summarized as: 1) the atmospheric $\left|\Delta m_{31}^{2}\right| \sim(1-5) \cdot 10^{-3} \mathrm{eV}^{2}$ is associated with a mixing, $\theta_{23}$, near to maximal [i]

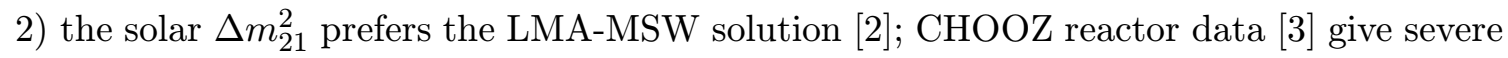
limits for $\left|U_{e 3}\right|$. In this contribution we are going to discuss that contrary to a wide spread belief, Earth effects on the propagation of atmospheric neutrinos can become observable [4] even if $\left|U_{e 3}\right|$ is small, but non-vanishing. This fact would allow to determine the sign of $\Delta m_{31}^{2}$ [i $\left[\bar{b}_{1}\right]$. For baselines $L$ smaller than the Earth diameter, appropiate for atmospheric neutrinos, $\frac{\Delta m_{21}^{2}}{4 E} L \equiv \Delta_{21} \ll 1$, so that we will neglect the (1,2)-oscillating phase in vacuum against the $(2,3)$-one. This is a very good aproximation, unless the high $\Delta m_{21}^{2}$-region of the LMA solution turns out to be the solution to the solar problem. In that case we should take into account corrections of order $O\left(\frac{\Delta m_{21}^{2}}{\Delta m_{21}^{2}}\right)$ (see eg.

In section 2 we discuss the correspondence between the determination of the sign of $\Delta m_{31}^{2}$ and the observation of the Earth effects in a transition involving $\nu_{e}$. The change expected in the neutrino spectrum and mixing due to matter effects, both for $\sin \theta_{13} \equiv$ $s_{13}=0$ and $s_{13} \neq 0$, are pointed out. In the latter case, section 3 studies the observability of the MSW-resonance, with a positive conclusion for baselines $L \gtrsim 7000 \mathrm{~km}$. Its impact on the survival probability, $\nu_{\mu} \rightarrow \nu_{\mu}$, is pointed out. Section 4 gives an analysis of the matterinduced CPT-odd asymmetry, together with the realistic charge-asymmetry expected for atmospheric neutrinos. In section 5 we present some conclusions.

\footnotetext{
${ }^{*}$ Speaker.

${ }^{\dagger}$ Jose.Bernabeu@uv.es

${ }^{\ddagger}$ Sergio.Palomares@uv.es
} 


\section{The neutrino spectrum in matter}

Current analyses leave us with two alternatives for the spectrum of the three active neutrino species, either hierarchical or degenerate.

The effective neutrino potential due to the charged current interaction of $\nu_{e}$ with the electrons in the medium is [i] $V \equiv \frac{a}{2 E}=\sqrt{2} G_{F} N_{e}$, so that the effective hamiltonian, in

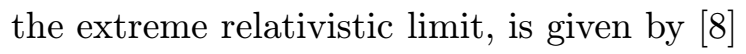

$$
H=\frac{1}{2 E}\left\{U\left(\begin{array}{ccc}
0 & 0 & 0 \\
0 & 0 & 0 \\
0 & 0 & \Delta m_{31}^{2}
\end{array}\right) U^{\dagger}+\left(\begin{array}{lll}
a & 0 & 0 \\
0 & 0 & 0 \\
0 & 0 & 0
\end{array}\right)\right\}
$$

In going from $\nu$ to $\bar{\nu}$, there are matter-induced CP- and CPT- odd effects associated with the change $a \rightarrow-a$. The additional change $\mathrm{U} \rightarrow \mathrm{U}^{*}$ is inoperative in the limit of $(\overline{2} \cdot \overline{1})$. The effects we are going to discuss depend on the interference between the different flavors and on the relative sign between $a$ and $\Delta m_{31}^{2}$. As a consequence, an experimental distinction between the propagation of $\nu$ and $\bar{\nu}$ (the sign of a) will determine the sign of $\Delta m_{31}^{2}$. An appreciable interference will be present if and only if there are appreciable matter effects. For atmospheric neutrinos, one needs the "connecting" mixing $U_{e 3}$ between the $\nu_{e}$-flavor and the $\nu_{3}$ mass eigenstate to show up.

For $s_{13}=0$, matter effects lead to a breaking of the (1,2)-degeneracy such that $\tilde{\nu}_{2}$ coincides with $\nu_{e}$. The net effect is that $\tilde{\nu}_{1}$ and $\tilde{\nu}_{3}$ in matter remain unaltered, i. e., as for vacuum $(2,3)$, leading to the $\nu_{\mu} \rightarrow \nu_{\tau}$ indicated by SK. The $\nu_{e}$-flavor decouples in matter, even if there was a large mixing in the $(1,2)$-system, as shown by the solar experiments. No matter effects would then be expected when starting with $\nu_{\mu}$. The CHOOZ limit [i]in, $\sin ^{2} 2 \theta_{13} \leq 0.10$, is it then fatal?

For small $s_{13}$, even if the effects on the spectrum are expected to be small, there could be a substantial mixing of $\nu_{e}$ with $\tilde{\nu}_{3}$ if one is near to a situation of level-crossing. This would lead to a resonant MSW behaviour [i.

$$
\sin ^{2} 2 \tilde{\theta}_{13}=\frac{4 s_{13}^{2} c_{13}^{2}}{\left(\alpha-\cos 2 \theta_{13}\right)^{2}+4 s_{13}^{2} c_{13}^{2}} \quad, \quad \alpha \equiv \frac{a}{\Delta m_{31}^{2}}
$$

But still $\left\langle\tilde{\nu}_{1} \mid \nu_{e}\right\rangle=0$, i. e., the $\nu_{e}$ has no overlap with the lowest mass eigenstate in matter. This vanishing mixing in matter is responsible for the absence of fundamental $\mathrm{CP}$-violating effects, even if there are three non-degenerate mass eigenstates in matter. In vacuum, the absence of genuine CP-odd probabilities was due to the degeneracy $\Delta_{21}=0$. The step from vanishing $\Delta_{21}$ in vacuum to the vanishing mixing $U_{e 1}$ in matter was termed a "transmutation" [i [10

For matter of constant density, there is no asymmetry associated with time-reversal $\mathrm{T}$, contrary to the matter-induced CP- and CPT- odd asymmetries. As a consequence, the T-conjugated transitions $(\alpha \neq \beta)$ have equal appearance probabilities [i] $\overline{1}_{1}^{1} \overline{1}_{1}, P\left(\nu_{\alpha} \rightarrow \nu_{\beta}\right)=$ $P\left(\nu_{\beta} \rightarrow \nu_{\alpha}\right)$. 


\section{Observability of the MSW resonance}

Non-resonant important matter effects are induced in the non-leading appearance channel governed by $\Delta_{31} \equiv \frac{\Delta m_{31}^{2}}{4 E} L$. The corresponding probability for $\alpha \neq 1$ is given by [i-i]

$$
P\left(\nu_{e} \rightarrow \nu_{\mu}\right) \simeq s_{23}^{2} \frac{4 s_{13}^{2}}{(1-\alpha)^{2}} \sin ^{2}\left[\Delta_{31}(1-\alpha)\right]
$$

This probability has a good sensitivity to $s_{13}$. Besides the change in the effective mixing, the interference pattern is modified to an oscillating phase, $\tilde{\Delta}_{31} \simeq \Delta_{31}(1-\alpha)$, which contains a baseline dependence as a combination of the standard $L / E$, plus the (constant) $\times L$ terms [i 100$]$. This is reminiscent of the Aharonov-Bohm effect, able to see the potential in quantum-mechanical phases.

For a baseline of $L=3000 \mathrm{~km}$, appropiate for neutrino factories [1] 201 , there are nonresonant Earth-matter effects in the sub-leading appearance probability. At the resonant energy $E_{R} \sim 9.6 \mathrm{GeV}$, there is no observable trace of the resonance. This suppresion can be understood because a resonant mixing is associated with level-crossing, so that $\Delta \tilde{m}_{31}^{2}$ is minimum on the resonance and then, $\tilde{\Delta}_{31} \simeq 0$ for such $L$.

For atmospheric $\nu_{\mu}$ neutrinos, matter effects in the survival probability $\nu_{\mu} \rightarrow \nu_{\mu}$ would be minute unless the resonance shows up. The resonance was not apparent even at $L=$ $3000 \mathrm{~km}$. Is there a way out?

Again, a non-vanishing connecting mixing $s_{13} \neq 0$ provides the solution. Along with it, there is a resonance width which, when discussed in terms of the dimensionless parameter $\alpha$, is given by

$$
\alpha_{R}=\cos 2 \theta_{13} \quad, \quad \Gamma_{\alpha}=2 \sin 2 \theta_{13}
$$

One discovers that the oscillating phase on the resonance is non-vanishing, but given by the $L$ dependent relation

$$
\tilde{\Delta}_{31(R)}=\Delta_{31} \frac{\Gamma_{\alpha}}{2}
$$

If $L \ll L_{o p t}$, with optimal $L, L_{o p t}$, defined by $\tilde{\Delta}_{31(R)}=\pi / 2$, the resonance does not affect the oscillation probability. On the contrary, around $L_{\text {opt }}=\frac{2 \pi}{\tilde{a} \tan 2 \theta_{13}}$, where $\tilde{a}=a / E$, the resonance be-
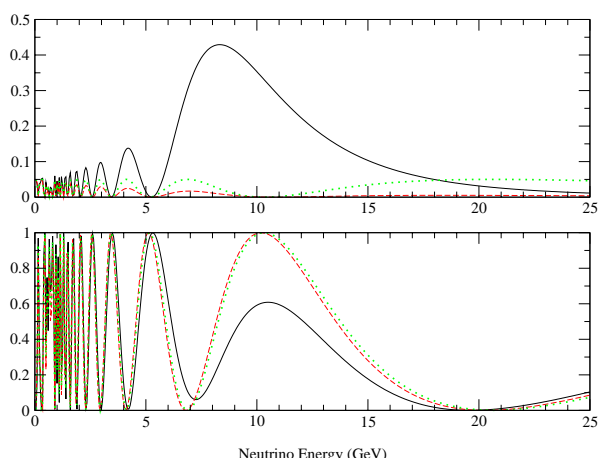

Figure 1: Appearance (upper panel) and survival (lower panel) probabilities, $P\left(\nu_{\mu} \rightarrow \nu_{e}\right)$ and $P\left(\nu_{\mu} \rightarrow \nu_{\mu}\right)$, for neutrinos (solid line), antineutrinos (dashed line) and vacuum (dotted line), for $L=8000$ $\mathrm{km}, \Delta m_{31}^{2}=3.2 \cdot 10^{-3} \mathrm{eV}^{2}, \sin ^{2} 2 \theta_{23}=1$ and $\sin ^{2} 2 \theta_{13}=0.1$. comes apparent and $L_{\text {opt }}$ is independent of $\Delta m_{31}^{2}$, which determines the resonant energy. For $L=L_{\text {opt }}$, the maximum mixing is accompanied by maximum oscillating factor.

Under these conditions, all channels would see the resonant effect. Contrary to nonresonant matter effects, the resonance only affects the (anti)neutrino channels if $\Delta m_{31}^{2}>$ $0(<0)$. For a baseline $L=8000 \mathrm{~km}$, this is shown in fig. $\varliminf_{1}^{1}$ for both $\nu_{e} \rightarrow \nu_{\mu}$ and $\nu_{\mu} \rightarrow \nu_{\mu}$ channels. 


\section{Charge asymmetries}

As discussed in section 3, matter effects distinguish neutrinos from antineutrinos. It is convenient to present them in terms of CP-odd (for appearance channels) and CPT-odd (for the survival probabilities) asymmetries. In the limit $\Delta_{21}=0$, there is no room for genuine $\mathrm{CP}$ violation. The interaction with matter will generate an asymmetry effect, however, which is not connected with the vacuum propagation.

For $\nu_{\mu}$ and $\bar{\nu}_{\mu}$, one has

$$
A_{C P T}=\frac{P\left(\nu_{\mu} \rightarrow \nu_{\mu} ; x\right)-P\left(\bar{\nu}_{\mu} \rightarrow \bar{\nu}_{\mu} ; x\right)}{P\left(\nu_{\mu} \rightarrow \nu_{\mu} ; x\right)+P\left(\bar{\nu}_{\mu} \rightarrow \bar{\nu}_{\mu} ; x\right)}
$$

and it is represented in fig. energy for a baseline of $L=8000 \mathrm{~km}$ and different values of $\sin ^{2} 2 \theta_{13}$. Around the resonance, $A_{C P T}$ presents a plateau with non-vanishing appreciable values (depending on $\sin ^{2} 2 \theta_{13}$ ). The big asymmetries at 6 and $20 \mathrm{GeV}$ correspond to low probabilities and they are not of interest. The negative (positive) asymmetry in the plateau is obtained for $\Delta m_{31}^{2}>0(<0)$. Obviously, it is symmetric with respect to the horizontal axis when changing the sign of $\Delta m_{31}^{2}$. As we have seen above, the optimal
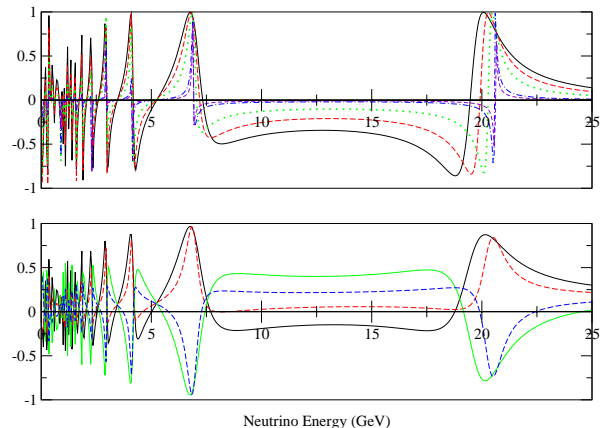

Figure 2: Upper panel: CPT-asymmetry, $A_{C P T}$ for different values of $\sin ^{2} 2 \theta_{13}$. From up to down: $\sin ^{2} 2 \theta_{13}=0.005,0.01,0.05,0.10,0.16$ and $\Delta m_{31}^{2}>0$ as all the plots are symmetric with respect to the horizontal axis when $\Delta m_{31}^{2}<0$. Lower panel: Chargeasymmetry, A, for $\sin ^{2} 2 \theta_{13}=0.05$ (dashed line) and 0.16 (solid line). The lower plots correspond to $\Delta m_{31}^{2}>0$ and the upper ones to $\Delta m_{31}^{2}<0$ For both panels, $L=8000 \mathrm{~km}, \sin ^{2} 2 \theta_{23}=1$ and $\left|\Delta m_{31}^{2}\right|=3.2 \cdot 10^{-3} \mathrm{eV}^{2}$. baseline is inversely proportional to the $\theta_{13}$ mixing.

For atmospheric neutrinos, $A_{C P T}$ cannot be separated out and the $\nu_{e}\left(\bar{\nu}_{e}\right)$ flux also contributes to the detection of $\nu_{\mu}\left(\bar{\nu}_{\mu}\right)$. Taking into account the CC cross-sections in the detector,

$$
\begin{aligned}
& N\left(\mu^{-} ; E\right)=\sigma_{c c}\left(\nu_{\mu}\right)\left[\phi^{o}\left(\nu_{\mu} ; E\right) P\left(\nu_{\mu} \rightarrow \nu_{\mu}\right)+\phi^{o}\left(\nu_{e}: E\right) P\left(\nu_{e} \rightarrow \nu_{\mu}\right)\right] \\
& N\left(\mu^{+} ; E\right)=\sigma_{c c}\left(\bar{\nu}_{\mu}\right)\left[\phi^{o}\left(\bar{\nu}_{\mu} ; E\right) P\left(\bar{\nu}_{\mu} \rightarrow \bar{\nu}_{\mu}\right)+\phi^{o}\left(\bar{\nu}_{e} ; E\right) P\left(\bar{\nu}_{e} \rightarrow \bar{\nu}_{\mu}\right)\right]
\end{aligned}
$$

where $\phi^{o}\left(\nu_{\mu} ; E\right)\left(\phi^{o}\left(\bar{\nu}_{\mu} ; E\right)\right)$ and $\phi^{o}\left(\nu_{e} ; E\right)\left(\phi^{o}\left(\bar{\nu}_{e} ; E\right)\right)$ are the muon and electron (anti) neutrino fluxes, respectively, calculated from [i] $\left.\overline{3}_{1}\right]$. As in the important energy range, both cross-sections are, to good aproximation, linear with the energy, one can build an asymmetry which eliminates what is induced by $\sigma_{c c}$ in the form

$$
A=\frac{N\left(\mu^{-} ; E\right)-\frac{\sigma_{c c}\left(\nu_{\mu}\right)}{\sigma_{c c}(\bar{\nu} \mu)} N\left(\mu^{+} ; E\right)}{N\left(\mu^{-} ; E\right)+\frac{\sigma_{c c}\left(\nu_{\mu}\right)}{\sigma_{c c}\left(\overline{\nu_{\mu}}\right)} N\left(\mu^{+} ; E\right)}
$$

In $\left(\overline{4} .3^{3}\right)$ there is still some asymmetry generated by the atmospheric neutrino fluxes. Contrary to $A_{C P T}$, the value of the muon-charge asymmetry is not symmetric with respect to the abscisa axis when changing the sign of $\Delta m_{31}^{2}$. In fig. for two values of $\sin ^{2} 2 \theta_{13}$. There is again an appreciable separation between the cases of positive and negative $\Delta m_{31}^{2}$. 


\section{Conclusions}

In the limit of $\frac{\Delta m_{21}^{2}}{4 E} L \ll 1$, the main conclusions of this study are: i) The medium effects, which discriminate between neutrino and antineutrino propagation determine the sign of the atmospheric $\Delta m_{31}^{2}$; ii) for $s_{13}=0$, electron neutrinos decouple from neutrino mixing in matter and have a definite effective mass in matter; iii) for $s_{13} \neq 0$, electron neutrinos mix with the third mass eigenstate neutrino and take part in the atmospheric neutrino oscillations; iv) electron neutrinos do not mix with the first mass eigenstate in matter, avoiding the generation of genuine $\mathrm{CP}$-violating effects; v) non-resonant medium effects are already apparent in the sub-sominant channel $\nu_{e} \rightarrow \nu_{\mu}$ for baselines $L \sim 3000 \mathrm{~km}$, in both the mixing and the oscillation phase-shift; vi) the observation of matter effects in the $\nu_{\mu}$-survival probability requires the action of the MSW resonance, with baselines longer than $L \sim 7000 \mathrm{~km}$; vii) the optimal baseline depends on the value os $s_{13}$, but the effects are much cleaner in the region of the longest baselines without entering the Earth core [i⿶凵] (nadir angles $\theta_{n} \gtrsim 33^{\circ}$ ).

This work is supported by Grant AEN-99-0296.

\section{References}

[1] H. Sobel, SuperKamiokande Collaboration, at Neutrino 2000, Sudbury, Canada (2000).

[2] Y. Suzuki, SuperKamiokande Collaboration, at Neutrino 2000, Sudbury, Canada (2000); Q. R. Ahmad et al., SNO Collaboration, 'P

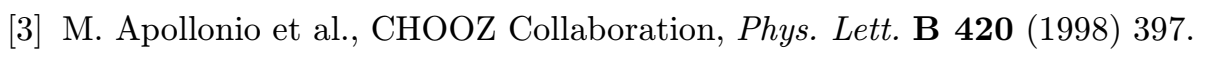

[4] M. C. Bañuls, G. Barenboim and J. Bernabéu, 'P hep-ph/0102184; J. Bernabéu, A. Pérez, S. Palomares-Ruiz and S. T. Petcov, hep-ph/0110071.

[5] I. Mocioiu and R. Schrock, JJ.High Energy Phys. 0111_(2001) 050; hep-ph/0106139; M. Freund, 'Phys. Rev D 64 4 20012053003 , hep-ph/0103300.

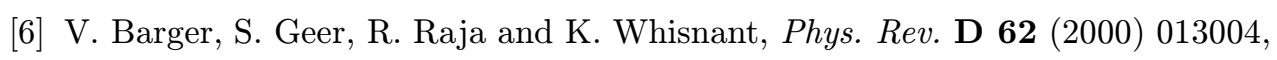
hep-ph/9911524.

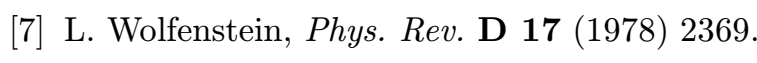

[8] T. K. Kuo and J. Pantaleone, Rev. Mod. Phys. $\mathbf{6} 1-1989) 93 \overline{1}$.

[9] S. P. Mikheyev and A. Yu. Smirnov, Sov. Ju Nucl. Phys. 42 1985$) 91 \overline{3}_{\mathrm{r}}$

[10] J. Bernabéu and M. C. Bañuls, 'Nucl. Phys. 87 (Proc. Suppl. 2 (2000) 315i', hep-ph/0003299.

[11] J. Bernabéu, Proc. WIN '99, p. 227; C. A. Domínguez and R. D. Viollier, Eds. World Scientific (2000), hep-ph/9904474.

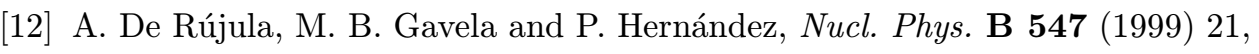
hep-ph/9911390; K. Dick, M. Freund, M. Lindner and A. Romanino, Nucl. Phys. B- $\overline{\mathbf{5}} \mathbf{6} \overline{2}$, [ -

[13] G. Fiorentini, V.A. Naumov and F.L. Villante, 'P 2 hys. Lett. B 510001 hep-ph/0103322; G. Fiorentini, V.A. Naumov and F.L. Villante, in the Proceedings of 27th International Cosmic Ray Conference, Hamburg, August 7-15, 2001, Vol.3, p.1218; hep-ph/0106014. 